\title{
Characteristics of patients with minor ischaemic strokes and negative MRI: a cross-sectional study
}

\author{
Fergus N Doubal, ${ }^{1}$ Martin S Dennis, ${ }^{1}$ Joanna M Wardlaw ${ }^{1,2}$
}

${ }^{1}$ Division of Clinical Neurosciences, Bramwell Dott Building, University of Edinburgh, Western General Hospital, Edinburgh, UK ${ }^{2}$ Scottish Imaging Network, A Platform for Scientific Excellence (SINAPSE) Collaboration, Scottish Funding Council Brain Imaging Centre, University of Edinburgh, Edinburgh, UK

\section{Correspondence to} Dr Fergus N Doubal, Division of Clinical Neurosciences, Bramwell Dott Building, University of Edinburgh, Western General Hospital, Edinburgh EH4 2XU, UK: fergus.doubal@ed.ac.uk

Received 28 July 2009 Revised 16 December 2009 Accepted 17 December 2009 Published Online First 27 June 2010

\section{(2) UNLOCK:}

This paper is freely available online under the BMJ Journals unlocked scheme, see http:// jnnp.bmj.com/site/about/ unlocked.xhtml.

\section{ABSTRACT}

Background Diffusion-weighted (DWI) MRI is recommended in UK guidelines to evaluate minor strokes, yet can produce negative results.

Objective The authors determined the rate of negative MRI (including DWI) and associated features in patients presenting to hospital with minor strokes.

Methods The authors performed a prospective observational cross-sectional study in a teaching hospital of patients with a clinical diagnosis of ischaemic lacunar or minor cortical stroke. The authors performed MRI (DWI, T2, fluid-attenuated inversion recovery (FLAIR), $\mathrm{T}^{*}$ and $\left.\mathrm{T} 1\right)$ as soon as possible after presentation. The authors used multivariate analysis to determine predictors of negative DWI and MRI (all sequences). Gold standard for clinical diagnosis of stroke was the opinion of an expert panel.

Results The authors recruited 246 patients, mean age 68.1 years (SD 11.6 years), 162 were males (66\%), and the median NIHSS was 2 (range 0 to 8). The median time from stroke onset to MR scan was 12 days (IOR 4-27 days). Eighty-one patients (33\%) did not show any ischaemia on DWI. Sixty patients (24\%) did not show the recent infarct on MRI (DWI/T2/FLAIR). With multivariate analysis, less severe stroke, younger age, female gender and increased time from stroke onset to scan were associated with negative DWI. With multivariate analysis, younger age and female gender were associated with negative MRI (DWI or T2 or FLAIR) scans.

Conclusions There is a high rate of negative MRI and DWl among patients with minor stroke (a third) which has important management and research implications. A negative MRI or DWI does not exclude the diagnosis of stroke.

\section{INTRODUCTION}

UK guidelines recommend MRI in patients presenting with minor stroke. ${ }^{2} 2$ Diffusionweighted MR imaging (DWI) is more sensitive than CT scanning at detecting acute cerebral ischaemia in minor stroke. ${ }^{3} 4$ DWI can be positive for ischaemia for several weeks after minor stroke, ${ }^{56}$ but there is also a negative rate for DWI in patients with clinically definite stroke. ${ }^{78}$ It is unclear which features are associated with negative DWI. We investigated the rate and associations of negative DWI and MRI in patients presenting with minor ischaemic stroke.

\section{METHODS}

We recruited patients prospectively between April 2005 and December 2007. We included patients presenting to our teaching hospital (which sees 550 patients/year with stroke) with a clinical diagnosis of minor ischaemic stroke (defined as lacunar or partial anterior circulation stroke $e^{9}$. We defined stroke if it was diagnosed by a panel of experts (neuroradiologist, vascular neurologist and stroke physician all blinded to the study hypothesis) with full access to MRI (to exclude other structural causes of the symptoms) and other investigation results. We excluded patients with transient ischaemic attacks (symptom duration $<24 \mathrm{~h}$ ), contraindications to MRI and those without a clinical diagnosis of minor ischaemic stroke. In August 2009, we reviewed all case records in those with negative imaging, to see if an alternative diagnosis had emerged, and re-reviewed the initial diagnosis of stroke. The follow-up period ranged from 21 to 52 months.

Patients had MRI after presentation on a $1.5 \mathrm{~T}$ MR scanner (Signa LX; General Electric, Milwaukee, Wisconsin) with $22 \mathrm{mT} \mathrm{m}^{-1}$ maximum strength gradients, including axial DWI, T2-weighted, fluidattenuated inversion recovery (FLAIR), T2* and sagittal T1 weighted sequences. A consultant neuroradiologist, blinded to the study hypothesis but not clinical information, coded acute relevant infarcts (DWI increased signal, reduced signal on apparent diffusion coefficient map, or increased signal with/without swelling without evidence of cavitation on FLAIR/T2) using a validated morphological schema. ${ }^{10}$

Patients had carotid Doppler ultrasound, electrocardiogram, routine blood tests and further clinical investigations as indicated. We defined time to scan as the time from stroke onset to scan, the persistence of symptoms was recorded at the time of the scan and age at presentation to hospital. We recorded stroke severity (NIHSS) and gender.

We defined minor cortical and lacunar stroke syndromes as previously (but did not use a subclassification system to diagnose stroke). ${ }^{11} \mathrm{We}$ further classified stroke subtype using radiological criteria, that is whether any recent infarct on MRI was cortical or lacunar, and used both clinical and radiological classification to subtype stroke. Where classifications differed, the radiological classification was used.

To determine which variables were included in the multivariate model, we used parametric and nonparametric tests as appropriate to assess univariate associations between variables and the presence of a negative scan. We used multivariate logistic regression to assess contributing factors with the binary outcome of positive/negative scan as the dependent variable. Calculations were performed with Minitab (version 15; Minitab, State College, Pennsylvania), and the $\alpha$ level for significance was 0.05 . We excluded 
one patient with an incomplete dataset. There were no suitable data for sample size calculations.

The Lothian Regional Ethics Committee approved this study. Patients provided written informed consent.

\section{RESULTS}

We screened 355 patients and recruited 253 patients. We excluded seven patients upon further review of case notes who did not have a clinical diagnosis of ischaemic stroke when final follow-up information was taken into account (three patients had functional disease, one patient had a TIA, and three patients had migraine) leaving 246 patients with definite stroke for analysis.

The mean age of those included was 68.1 years (SD 11.6 years). There were 162 males (66\%), and the median NIHSS score was 2 (range $0-8$ ). The median time from stroke onset (not presentation) to MR scan was 12 days (IOR 4-27 days).

Of 246 patients, 81 (33\%) had no ischaemia on DWI, and 60 (24\%) did not show the recent infarct on any MRI sequence (DWI/T2/FLAIR). In 21 patients, there was a recent symptomatic infarct seen on T2/FLAIR not seen on DWI. With a univariate analysis, there was no association between lacunar stroke and negative DWI or MRI (T2/FLAIR/DWI) scan, and this was excluded from the multivariate model.

With multivariate analysis, a negative DWI was associated with younger age, lower NIHSS, female gender and increased time from stroke onset to scan after correcting for each other and the presence of persisting weakness. The odds of having a negative scan increased by $2 \%$ (95\% CI 1 to $3 \%$ ) for each additional day lapsed between stroke and scan (OR 1.02). The proportion of patients with negative DWI for those scanned between 0 and 6 days was $12 \%, 7-13$ days $30 \%, 14-21$ days $34 \%$ and $>21$ days $56 \%$.

With a multivariate analysis, a negative MRI (DWI/ FLAIR/T2) was associated with younger age and female gender after correcting for each other and time to scan, the presence of persisting symptoms and NIHSS (table 1).

\section{DISCUSSION}

In patients presenting with minor ischaemic stroke in the anterior circulation, 33\% had negative DWI, and $24 \%$ had negative MRI (DWI/T2/FLAIR). Negative DWI was associated with increasing time between stroke and scan, less severe stroke, younger age and female gender after correcting for other factors. Negative MRI (DWI/T2/FLAIR) was associated with younger age and female gender.

This study has several strengths: all patients had a final clinical diagnosis of stroke made by an experienced stroke team, with diagnostic MRI and follow-up to see if an alternative diagnosis had emerged in the patients with negative scans. We used a dedi- cated MR research scanner with standardised procedures with scans coded by an experienced neuroradiologist. These results are generalisable to patients presenting with clinical features and routine investigations suggestive of a minor ischaemic stroke.

There are some weaknesses. Despite our best efforts and reference panel discussion, some of the subjects may have had a stroke mimic, especially as some of the negative scans were in younger females whose symptoms were compatible with migraine or functional disease. Previous studies have confirmed a diagnosis of stroke in patients with initially negative DWI with infarcts on follow-up scans, ${ }^{12}$ but some patients may never develop a relevant visible lesion. This was a cross-sectional study, so we can only report associations. The duration of DWI lesions in longitudinal studies after stroke demonstrates a high interindividual variability, ${ }^{6}$ and we cannot comment on how long DWI lesions remain on an individual basis. New asymptomatic DWI lesions may appear after ischaemic stroke. ${ }^{13}$ We only reported a DWI lesion as positive if it was in an arterial territory relevant to the most recent symptoms. Our results do not generalise to patients with posterior circulation stroke. If stroke awareness and services improve, the time from stroke symptoms to scan will decrease. The generalisability of our results may be limited by the relatively late median time to scan of 12 days in this mainly outpatient population, attributable to delays in patients presenting to hospital.

How do our results compare with other studies? A similar rate of negative DWI (30\%) is reported in patients with minor stroke scanned at a median of 17 days poststroke, ${ }^{8}$ but this study did not distinguish between stroke and TIA in the multivariate regression. Studies of DWI within $24 \mathrm{~h}$ of acute stroke found negative DWI rates ranging from $6 \%{ }^{12}{ }^{14}$ to $17 \%{ }^{4}$ but were retrospective ${ }^{12}$ or reclassified patients presenting with TIA but who had an acute ischaemic lesion on DWI as stroke. ${ }^{4}$ Other studies did not report negative DWI rates in TIA and stroke separately. ${ }^{15}$

Lower NIHSS (lower stroke severity) was associated with negative DWI. In general, small infarcts are less likely to be identified on imaging than large infarcts, and although infarct size was shown in a small longitudinal study not to predict the duration of DWI-visible lesions, ${ }^{6}$ it is probable that small infarcts resolve quicker than larger infarcts.

Increased time to imaging was associated with negative DWI although not negative T2/FLAIR MRI. This confirms other findings ${ }^{8}$ and is attributable to normalisation of the DWI signal as cellular oedema resolves, but this does not mean that the tissue damage has resolved. Consistent with this, we have demonstrated that T2/FLAIR identifies more infarcts than DWI in patients presenting subacutely with minor stroke. Female gender was associated with both negative DWI and negative T2/FLAIR MRI. Gender did not previously predict negative DWI in patients with TIA/minor stroke combined. ${ }^{8}$ The cause of this

Table 1 Multivariate associations of a negative diffusion-weighted imaging (DWI) and MRI (T2 or fluid-attenuated inversion recovery (FLAIR) or DWI)

\begin{tabular}{|c|c|c|c|c|}
\hline \multirow[b]{2}{*}{ Variable } & \multicolumn{2}{|l|}{ Negative DWI } & \multicolumn{2}{|c|}{ Negative MRI (T2/FLAIR/DWI) } \\
\hline & OR (95\% CI) & p Value & OR (95\% CI) & p Value \\
\hline Age (years) & $0.97(0.95$ to 1.00$)$ & 0.047 & 0.96 (0.93 to 0.98$)$ & 0.001 \\
\hline Male sex & $0.44(0.24$ to 0.81$)$ & 0.008 & $0.46(0.24$ to 0.87$)$ & 0.018 \\
\hline $\begin{array}{l}\text { Persisting symptoms at time of scan } \\
\text { (yes/no) }\end{array}$ & $0.61(0.29$ to 1.28$)$ & 0.191 & $0.50(0.23$ to 1.08$)$ & 0.077 \\
\hline
\end{tabular}

The odds ratios (ORs) are per unit increase in the explanatory variable, and all ORs are corrected for all explanatory variables in the table. 
discrepancy is not clear; possibly, our result was due to residual confounding from baseline imbalances.

We have shown that a third of patients presenting with minor stroke have negative DWI, which is associated with decreased stroke severity, increased time to scan, younger age and female gender. Had we been less critical with our clinical diagnosis of stroke and included even some of the 355 patients with suspected stroke that were initially screened, the proportion with negative DWI would have been higher. These results should alert clinicians about when to expect a negative MRI in a patient with minor stroke and encourage them not to automatically exclude the diagnosis of stroke if DWI is negative.

Acknowledgements Statistical advice was from C Graham, Statistician, Wellcome Trust Clinical Research Facility, Edinburgh, UK.

Funding FD was funded by the Wellcome Trust (075611). The Chief Scientists Office (Scotland) funded the brain imaging (CZB-4-281), which took place in the SFC Brain Imaging Research Centre (http://www.sbirc.ac.uk), a member of the SINAPSE Collaboration (Scottish Imaging Network, A Platform for Scientific Excellence, (http://www.sinapse.ac.uk). JMW is part funded by the Scottish Funding Council through the SINAPSE Collaboration.

Competing interests None.

Patient consent Obtained.

Ethics approval Ethics approval was provided by the Lothian Regional Ethics Committtee, UK.

Contributors FND conceived the study, collected and analysed data and wrote the paper. MSD helped conceive the study, collected data and commented on drafts of the paper. JMW helped conceived the study, obtained original study funding, analysed all MRI scans and commented on drafts of the paper. All authors had full access to all of the data and take responsibility for the integrity of the data and the accuracy of the data analysis.

Provenance and peer review Not commissioned; externally peer reviewed.

\section{REFERENCES}

1. Department of Health. National Stroke Strategy. London: DH, 2007. http://www. dh.gov.uk/prod_consum_dh/groups/dh_digitalassets/documents/digitalasset/ dh_081059.pdf.

2. Scottish Intercollegiate Guidelines Network (SIGN). Management of patients with stroke or TIA: Assessment, Investigation, Immediate Management and Secondary Prevention. Guideline 108. http://www.sign.ac.uk/guidelines/fulltext/108/index.html (accessed Jul 2009).

3. Brazzelli M, Sandercock PAG, Chappell F, et al. Magnetic resonance imaging versus computed tomography for detection of acute vascular lesions in patients presenting with stroke symptoms. Cochrane Database Syst Rev 2009; (4):CD007424.

4. Chalela JA, Kidwell CS, Nentwich LM, et al. Magnetic resonance imaging and computed tomography in emergency assessment of patients with suspected acute stroke: a prospective comparison. Lancet 2007;369:293-8.

5. Keir S, Wardlaw JM, Bastin ME, et al. In which patients is diffusion-weighted magnetic resonance imaging most useful in routine stroke care? J Neuroimaging 2004;14:118-22

6. Schulz UGR, Flossmann E, Francis JM, et al. Evolution of the diffusion-weighted signal and the apparent diffusion coefficient in the late phase after minor stroke. $J$ Neurol 2007;254:375-83.

7. Ay H, Buonanno FS, Rordorf G, et al. Normal diffusion-weighted MRI during strokelike deficits. Neurology 1999;52:1784-92.

8. Schulz UG, Briley D, Meagher T, et al. Diffusion-Weighted MRI in 300 Patients Presenting Late With Subacute Transient Ischemic Attack or Minor Stroke. Stroke 2004;35:2459-65.

9. Bamford J, Sandercock P, Dennis M, et al. Classification and natural history of clinically identifiable subtypes of cerebral infarction. Lancet 1991;337:1521-6

10. Wardlaw JM, Sellar RJ. A simple practical classification of cerebral infarcts on CT and its interobserver reliability. Am J Neuroradiol 1994;15:1933-9.

11. Doubal FN, Dhillon B, Dennis MS, et al. Retinopathy in ischemic stroke subtypes. Stroke 2009:40:389-93.

12. Oppenheim C, Stanescu R, Dormont D, et al. False-negative diffusion-weighted MR findings in acute ischemic stroke. Am J Neuroradiol 2000;21:1434-40.

13. Kang DW, Latour $\mathrm{LL}$, Chalela JA, et al. Early ischemic lesion recurrence within a week after acute ischemic stroke. Ann Neurol 2003;54:66-74.

14. Mullins ME, Schaefer PW, Sorensen AG, et al. CT and conventional and diffusionweighted MR imaging in acute stroke: Study in 691 patients at presentation to the emergency department. Neuroradiology 2002;224:353-60.

15. Sylaja PN, Coutts SB, Krol A, et al. When to expect negative diffusion-weighted images in stroke and transient ischemic attack. Stroke 2008;39:1898-900. 